\title{
Moderate-to-Vigorous Physical Activity Levels in Physical Education, School Recess, and After-School Time: Influence of Gender, Age, and Weight Status
}

\author{
Jesús Viciana, Daniel Mayorga-Vega, and Alejandro Martínez-Baena
}

\begin{abstract}
Background: Primary and secondary school ages have been considered key moments to address the decrease of moderate-tovigorous physical activity (MVPA). Individual (eg, age, gender, and weight status) and contextual factors (moments of the day) need to be considered for a better explanation of the phenomenon. The quantity and quality of physical activity in Physical Education (PE), school recess (SR), and after school (AS) time need to be taken into account to solve the low levels of MVPA in youth. Methods: A sample of adolescents $(\mathrm{N}=231,14.6 \pm 1.2$ years old $)$ was studied using accelerometry to determine the objective MVPA level in PE, SR, and AS. Results: Results indicated statistically significant differences on MVPA between contexts $(\mathrm{AS}>\mathrm{PE}>\mathrm{SR}, P<.001)$ as well as regarding the individual factors: age (older $>$ younger in PE and younger $>$ older in SR time; $P<.001$ ), gender (boys $>$ girls in all contexts, $P<.001$ ), and weight status (overweight $>$ nonoverweight in AS, $P$ $<.01)$. Conclusions: Because students did not meet the daily MVPA recommendations, some strategies have been provided in each of the contexts analyzed.
\end{abstract}

Keywords: accelerometry, health behavior, adolescent, overweight, physical education

Currently most of the population does not achieve the daily recommendation of moderate-to-vigorous physical activity (MVPA) levels. ${ }^{1}$ Primary and secondary school ages have been considered key moments to address this problem because of the decrease in MVPA starting at this point (especially in adolescence) ${ }^{2}$ and the consequences of this decrease are manifested along the life span. ${ }^{3}$ Consequently, the quantity and quality of physical activity (PA) in Physical Education (PE), school recess (SR), and after school (AS) time are the main 3 contexts that researchers need to take into account to solve the low levels of MVPA in adolescents.

PA is a multidimensional and complex behavior that tends to vary considerably between students depending on individual factors. ${ }^{4}$ Therefore, to promote good levels of PA among adolescents, it is crucial to determine the influence of personal factors on PA in different school-based contexts to engage in the recommended MVPA. ${ }^{5}$ Among others, gender, age, and weight status are 3 of the main personal factors that influence levels of MVPA.

Some previous studies have separately examined PA levels during PE lessons, SR periods, and AS time among children and adolescents, as well as the influence of individual factors, such as gender, age, and weight status, in all of these settings. ${ }^{5-8}$ Different studies have been carried out analyzing 1 of these 3 contexts or combining 2 of them, as well as some of the individual factors previously mentioned. Results have been provided in relation with the minutes of MVPA in each context analyzed and with their contribution to the daily recommendations of MVPA $(60 \mathrm{~min} / \mathrm{d}){ }^{9}$

Related to the PE setting, various studies have obtained divergent results, some reaching the recommendations of $50 \%{ }^{10}$ of the

Viciana and Mayorga-Vega are with the Faculty of Sport Sciences, University of Granada, Granada, Spain. Martínez-Baena is with the Faculty of Physical Activity and Sport Sciences, University of Valencia, Valencia, Spain. Viciana (jviciana@ugr.es) is corresponding author. lesson's time involved in MVPA ${ }^{11,12}$ and others not. ${ }^{13,14}$ The majority of the studies confirmed the importance of PE in the total time of MVPA accumulated. ${ }^{15}$ In the SR period, results have shown that children and adolescents are involved in MVPA about 20\% to $30 \%$ of the time, ${ }^{16,17}$ which approximates $4 \%$ of the daily recommended MVPA. In some exceptions, such as Portugal where the school schedule is very long with various SR periods during the day, SR periods contribute in a greater extent to the total amount of the daily recommended MVPA (about 40\%). ${ }^{18}$ Finland, for instance, also has 30 SR periods a week of 10 minutes each for elementary schoolchildren, which supposes 300 minutes of SR time available for PA (double that of Spanish schoolchildren). ${ }^{19} \mathrm{AS}$ is the third segment of a normal day for students where MVPA could be increased. Results of this period are also contrasting and variable depending on multiple factors (eg, gender, age, parental restrictions, etc.), ${ }^{20}$ but it seems to be the most important period of the day regarding the contribution to MVPA recommendations. ${ }^{21}$ Moreover, organized sports activities seem to be crucial during this period to increase MVPA levels. ${ }^{22,23}$

Regarding the individual factors, there is more of a consensus between researchers that gender and age influence MVPA, being that younger boys are more active, and older girls are the most inactive. ${ }^{2,12}$ However, weight status has also had contrasting results. For instance, the study of Gao et $\mathrm{al}^{6}$ with middle school students between 10 to 14 years of age obtained significant differences in MVPA levels in favor of normal weight students rather than overweight and obese students. However, Hohepa et $\mathrm{al}^{7}$ found the opposite results with New Zealand adolescents, as those classified as overweight/obese were more active than the normal weighted adolescents. Despite these results, other studies found a nonrelationship between weight status and MVPA, such as the Spanish AFINOS (Actividad Física como prevención de Infecciones, Obesidad y Sobrepeso) study carried out with adolescents in the SR period by Martínez-Gómez et $\mathrm{al}^{24}$ the study performed by Hesketh et $\mathrm{al}^{25}$ with Australian students, or the study carried out by Mayorga-Vega and Viciana. ${ }^{26}$ 
To our knowledge, the study of Mayorga-Vega and Viciana ${ }^{26}$ is the only 1 that examines objectively the levels of MVPA in the 3 contexts (PE lessons, SR period, and AS time) within the same sample. However, the participants of their study were selected from adolescents who participated voluntarily in extracurricular sports activities, comparing the levels of MVPA in PE lessons, SR period, and these organized sports activities. This caused a self-selected sample that was more motivated toward PA than the normal population. Therefore, the results of this study should be only generalized to active adolescents. Unfortunately, to our knowledge there are no studies comparing the adolescents' objective MVPA levels between PE lessons, SR periods, and AS time, as well as examining the influence of gender, age, and weight status in each situation among the same sample and without restrictions. This information potentially can inform future policy concerning the promotion of the recommended MVPA levels in these important school-based opportunities. $^{5}$

Consequently, the purposes of this study were (1) to compare the objective MVPA levels between PE lessons, SR periods, and AS time in secondary school students and (2) to examine the influence of gender, age, and weight status on adolescents' objective MVPA levels during PE lessons, SR periods and AS time.

\section{Methods}

\section{Participants}

A sample of 231 Spanish secondary school students (age $=14.6 \pm$ 1.2 years, body mass $=58.1 \pm 12.3 \mathrm{~kg}$, body height $=163.0 \pm 8.6$ $\mathrm{cm}$, and body mass index $[\mathrm{BMI}]=21.7 \pm 3.6 \mathrm{~kg} / \mathrm{m}^{2}$ ) participated in the current study. From the initial sample of 345 (190 boys and 155 girls) who agreed to participate in the study, 114 were eliminated due to not fulfilling 1 or more of the following inclusion criteria: (1) recording 2 complete $\mathrm{PE}$ classes in a week with the accelerometer and (2) registering at least 2 of the $5 \mathrm{SR}$ and AS time periods of the school week with the accelerometer (the average of the SR periods and the AS time periods were considered for the study). All students and their parents or legal guardians were informed and gave their consent to participate in this research.

\section{Measures}

MVPA Levels. Objectively, MVPA was measured with a GT3X+ accelerometer (ActiGraph, LLC, Pensacola, FL) that has a high level of reliability and has been validated in adolescents. ${ }^{27}$ The accelerometer registers and measures variations of acceleration in a range of $\sim 0.95$ to 2.50 Gs with 3 axes that collect information and calculate the magnitude vector. The output data are digitalized by an analogic-digital converter of 12 bits and $30 \mathrm{~Hz}$ that digitally filters the signal, limiting the frequency to between 0.25 and 2.50 $\mathrm{Hz}$. The researcher decides the period, or epoch, in which the data will be periodically registered (each $1,5,15,30$ or 60 seconds). In the current study, the data were registered every second. ${ }^{28}$ The counts obtained in a particular epoch are proportional to the intensity of the PA during the measured period. ${ }^{29}$ To calculate the MVPA, the criteria of 2296 to 4011 for moderate PA and 4012 or more for vigorous PA were used. ${ }^{29}$ The data were treated with the ActiLife Lifestyle Monitoring System (software version 6.9.2).

Weight Status. Participants' weight status was categorized as nonoverweight and overweight (including obese), according to the BMI international cutoff values. ${ }^{30}$ For this purpose, participants' body weights and heights were previously measured and then BMI was calculated as body weight/height squared $\left(\mathrm{kg} / \mathrm{m}^{2}\right)$. Body mass and height were measured in shorts, T-shirts, and barefoot. For the body mass measure, the participant stood in the center of the scale (Seca, Ltd, Hamburg, Germany; accuracy $=0.1 \mathrm{~kg}$ ) without support and with the weight distributed evenly on both feet. For the body height assessment, the student stood with the feet together, with the heels, buttocks, and upper part of the back touching the scale (Holtain Ltd, Crymmych, Pembs, United Kingdom; accuracy = 0.1 $\mathrm{cm})$ and with the head placed in the Frankfort plane. The average of 2 measurements for both body mass and height was retained.

\section{Procedure}

The protocol of the current study was first approved by The Ethical Committee of the University of Granada. Then, the principal and the PE teacher of a secondary school center were contacted by a researcher to inform them about the project and to ask for their permission to conduct this study. After the school approval was obtained, students and their legal guardians were fully informed about all of the features of the study, and written informed consent to take part in the research was obtained from all participants' legal guardians. Several schools of the city center of Granada were considered for carrying out this research, but in the end only 1 school was selected because of the difficulty of coordinating the data collection in more than 1 school. The selected school was based on criteria that facilitated the development of the study, such as the number of students belonging to the secondary level of education, number of students by classroom, predisposition of legal guardians to collaborate with the research, or availability and willingness of the PE teacher to collaborate with the study.

Two trained researchers conducted anthropometric measurements and fitted accelerometers during the months of March to June of 2014. A first PE lesson was used for explaining the aim of the study, the rules (wearing the accelerometer during the entire day from when they wake up, to taking off the accelerometer only when doing aquatic activities or taking a shower), and the confidentiality of the data. Then the accelerometers were fitted, and students wore them during an entire week. Students were asked to continue with their normal activities during the week and were encouraged to continue with organized sports activities. PE lessons were conducted as usual, following the program of the subject designed by the teacher. In the case of this study, the students were developing technical and tactical abilities in basketball and small-sided games in all class groups, standardizing the PE lessons in all groups. Thus, the structure of the PE sessions during measures were as follows: 10 minutes of warm-up exercises, 5 to 10 exercises focused on tactical abilities during small-sided games (ie, $2 \times 2$ and $3 \times 3$ games), and 10 minutes of flexibility and relaxing exercises at the end of the session.

The requirements for being included in the study were to complete 60 minutes of the 2 PE classes in the week, at least 2 of the 5 SR periods during the weekdays, and at least 2 of the 5 complete periods of the AS time (from the end of the school schedule to the end of the day, from 14:00 to 00:00 h).

\section{Statistical Analyses}

Descriptive statistics (mean and SD) for all the variables were calculated. A 1-way analysis of variance (ANOVA) was used to examine the differences on MVPA levels between PE, SR, and AS sessions. Subsequently, post hoc with the Bonferroni adjustment was used to compare pairs of means. Afterward, an analysis of covariance (ANCOVA) of 2 groups (boys, girls) $\times 3$ contexts $(\mathrm{PE}$, $\mathrm{SR}, \mathrm{AS})$, with age and BMI values as covariates, was used to test 
the influence of gender on the MVPA levels. Subsequently, post hoc with the Bonferroni adjustment was used for both between or within-groups pairwise comparisons. Similarly, to examine the influence of age and weight status on MVPA levels, the other 2 factor values (eg, gender and BMI, and gender and age, respectively) were used as covariables. Beforehand, participants' age was categorized as younger (12.5 to 13.5 years) and older (14.5 to 15.5 years) and weight status was established as nonoverweight (BMI < overweight cut-point) and overweight (BMI $\geq$ overweight cut-point). ${ }^{30}$ Effect sizes were estimated using the partial eta squared $\left(\eta_{p}^{2}\right)$. All statistical analyses were performed using the SPSS Version 20.0 for Windows (IBM Corporation, Armonk, NY). The statistical significance level was set at $P<.05$.

\section{Results}

Means, SDs, and ANOVA results for MVPA levels under each situation for the whole sample, as well as ANCOVA results for gender, age, and weight status categories, are represented in Table 1. The 1-way ANOVA result indicated statistically significant differences on MVPA levels between PE, SR, and AS periods $\left(F_{2,460}=805.928\right.$, $\left.P<.001, \eta_{\mathrm{p}}^{2}=0.778\right)$. The post hoc pairwise comparison with the Bonferroni adjustment showed statistically significant higher MVPA values in AS time than in PE and SR and higher MVPA values in $\mathrm{PE}$ than in SR $(P<.001)$.

\section{Gender}

The 2-way ANCOVA result indicated a statistically significant interaction effect on MVPA levels $\left(F_{2,454}=14.970, P<.001, \eta^{2}\right.$ p $=0.062)$. Subsequently, the post hoc between-group pairwise comparisons with the Bonferroni adjustment showed statistically significant higher values for boys in PE, SR, and AS periods $(P<$ $.001)$. Regarding the within-group pairwise comparisons, both boys and girls showed statistically significant greater MVPA levels in AS time than in PE and SR and higher MVPA values in PE than in $\mathrm{SR}(P<.001)$.

\section{Age}

The 2-way ANCOVA result indicated a statistically significant interaction effect on MVPA levels $\left(F_{2,454}=4.047 ; P=.039 ; \eta_{\mathrm{p}}^{2}=0.018\right)$. Then, the post hoc between-group pairwise comparisons with the Bonferroni adjustment showed statistically significant higher values for older students in $\mathrm{PE}$, but higher values for younger students in SR $(P<.001)$. However, during the AS period statistically significant differences were not found between age categories $(P>.05)$. Regarding the within-group pairwise comparisons, both younger and older adolescents showed statistically significant greater MVPA levels in AS time than in PE and SR and greater MVPA levels in $\mathrm{PE}$ than in $\mathrm{SR}(P<.001)$.

\section{Weight Status}

The 2-way ANCOVA result indicated a statistically significant interaction effect on MVPA levels $\left(F_{2,454}=7.521, P=.004, \eta^{2}\right.$ p $=0.032$ ). Subsequently, the post hoc between-group pairwise comparisons with the Bonferroni adjustment showed statistically significant higher values for overweight adolescents in AS time $(P<$ $.01)$. However, during PE and SR statistically significant differences were not found between weight status categories $(P>.05)$. Regarding the within-group pairwise comparisons, both nonoverweight and overweight adolescents showed statistically significant greater MVPA levels in AS time than in PE and SR and greater MVPA levels in PE than in SR $(P<.001)$.

In addition, only $41 \%$ of the sample attained the daily recommendations of MVPA (57\% men and 24\% women; $42 \%$ younger and $40 \%$ older; $47 \%$ nonoverweight and $51 \%$ overweight).

\section{Discussion}

The first purpose of this study was to compare the objective MVPA levels between PE lessons, SR periods, and AS time in secondary school students. First, the adolescents studied did not achieve the recommendations of daily practice of MVPA (58 minutes, adding the 3 moments measured in PE, SR, and AS periods). ${ }^{9}$ Results revealed that the entire sample showed higher MVPA levels in AS time than in PE and SR and higher MVPA levels in PE than in SR. However, taking into account the total time considered for each of the periods (600 minutes for AS time, 30 minutes for SR, and 60 minutes for PE), PE was the period where the adolescents were involved in a higher percentage of MVPA (26.7\%) regarding the total time for the lesson, followed by SR period (10.8\%) and AS $(7.3 \%)$. Various studies agree on the importance of structured periods of PA as the better option in developing MVPA in children and adolescents (eg, PE lessons or organized sports activities AS), ${ }^{26,31}$ mainly due to the direction of a qualified expert. On the contrary, the time that depends on the individuals to practice MVPA (eg, SR time or free time during the AS range of time) is usually spent in sedentary habits, especially in critical moments of youth like in adolescence. ${ }^{2,32}$ In line with this, our results showed that PE lessons contributed efficiently to the daily recommended MVPA, as obtained by previous research. ${ }^{10,11}$

Unfortunately, the time involved in MVPA did not achieve the recommended $50 \%$ of the total PE lesson. ${ }^{10}$ Although the comparison of minutes the students were involved in MVPA during $\mathrm{PE}$ lessons is complex due to the differences in the duration of the PE sessions, different international studies have obtained distinct results, some achieving the recommended time ${ }^{11,12}$ and others not. ${ }^{13,14}$ Specific intervention programs during $\mathrm{PE}$ classes have been effective compared with usual practice, increasing by $24 \%$ the time involved in MVPA by students. ${ }^{33}$ Therefore, the first suggestion of this study is to promote different interventions to achieve the recommended $50 \%$ of the time in PE lessons. As Lonsdale et $\mathrm{al}^{33}$ concluded, further high-quality studies are necessary to identify which theoretical framework and psychosocial mediators positively influence the increment of MVPA during PE lessons.

The total MVPA time in SR time was scarce in the sample used in this study, barely $11 \%$ of the total time of the SR period, which is $4.4 \%$ of the daily recommendations of MVPA, in contrast to the $20 \%$ to $30 \%$ found in previous studies. ${ }^{11,12}$ Two main arguments found in the literature could be relevant to the results of this study: (1) Andersen et $\mathrm{al}^{34}$ found that the surface of the recreation area influences the amount of time students are involved in MVPA during the SR time. Grass and playground were good areas to promote PA, whereas solid surface areas had the highest proportion of time spent sedentary. (2) Watson et al, ${ }^{35}$ in a study with Australian girl students, found that the student uniform was a barrier for being involved in PA during SR. In the school of our study, these 2 factors could influence the MVPA obtained, due to the fact that in the majority of Spanish schools, the SR area had a solid surface (concrete), and, moreover, boys and girls of this school wore uniforms, except those who had a PE class in their schedule that day. A third argument mentioned by Uys et $\mathrm{al}^{36}$ that could have influenced as well is the crowdedness 


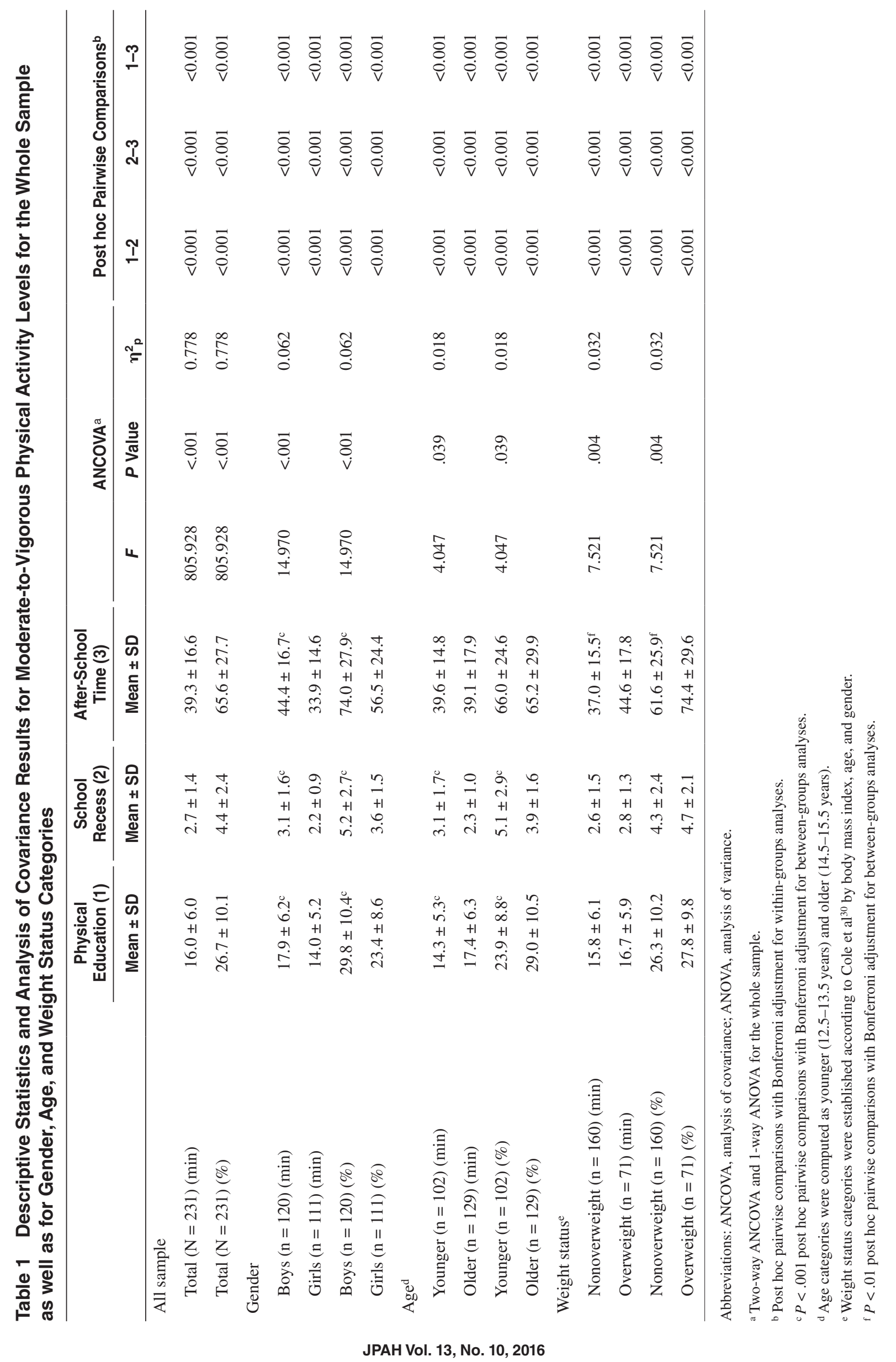


of the recreational area during particular hours of the SR time in the school of this study. Incrementing PA during this SR period seems crucial to increasing the contribution of school time to the daily recommendations of MVPA in adolescents. Strategies like the Sport Education Model carried out in the SR time (season phase) could be a good contribution to this increment. The motivation and the high indices of participation that suppose the competitions of this model for students ${ }^{37}$ may also entail more time involved in MVPA during SR time.

Regarding the AS time, it was the period that contributed more to the daily recommendations of MVPA at 65\% (almost 40 minutes). These results are in line with previous studies in relation to the importance of this period to the daily recommendations of MVPA, as demonstrated the systematic review carried out by Brooke et al. ${ }^{38}$ Nevertheless, the comparison between studies is complex due to the definition of the AS period, which has been considered in many ranges of hours (eg, from 16:00 to 18:00 $\mathrm{h}^{26}$ or from 15:30 to $20: 30$ h). ${ }^{39}$ The current study has defined the AS period as "the rest of the day" or extracurricular time (from the finalization of the school schedule until the end of the day) for the analysis of the data, which impacts the total quantity of MVPA obtained. Moreover, almost all of the adolescents in the current study participated in organized sports activities during their free time $(82.5 \%$ of the sample), which could influence the high MVPA level obtained. In general, previous studies have confirmed a drop in the MVPA time throughout the years during the AS period, ${ }^{21}$ the total decrease being quantified as approximately $7 \%$ by year in adolescence. ${ }^{40}$ Therefore, taking into account these previous data, promoting PA during AS time is recommendable. Different programs for the promotion of PA in leisure time have been successful. ${ }^{32}$ However, some authors commented that individuals are not willing to be involved in MVPA for a longer period than they usually are. Consequently, these authors suggested other forms of PA to increase the PA during this period as the utilitarian transportation. ${ }^{41}$ The use of exergames could also be an effective strategy to increase MVPA in $\mathrm{PE}$, as demonstrated in leisure time by Gao et al. ${ }^{42}$ Moreover, the meta-analysis of Plotnikoff et $\mathrm{al}^{43}$ emphasized the importance of social-cognitive theories and psychological factors (eg, perceived behavioral control, barriers, safety, environmental conditions, or self-efficacy) as future research lines to determine which factors influence MVPA in individuals' free time.

The second purpose of the study was to examine the influence of gender, age, and weight status on adolescents' objective MVPA levels during PE lessons, SR periods, and AS time. The results confirmed previous findings obtained in the majority of the studies regarding gender, where boys were involved in MVPA more than girls in each of the contexts analyzed. , $7,8,44$ However, some of these studies were carried out with questionnaires (not with an objective measurement of PA) or by addressing 1 or 2 of the contexts analyzed in the current study, but not all 3 of them.

In regards to age, significant differences were detected between older and younger students, varying depending on the context analyzed. In PE lessons older students were more active than younger, in SR time younger students were involved more in MVPA than older students, and in AS time no differences were found. On 1 hand, these results revealed that the relationship between age and MVPA is complex and depends on each context. In structured periods like PE lessons, MVPA could depend on some other factors associated to age, for instance, 1 possibility is the sense of responsibility of older students when an expert adult directs the activity. In the recent longitudinal study carried out by Brooke et al, ${ }^{45}$ statistical differences in MVPA were found between younger and older English students during the majority of segments in the day (in favor of younger students), but not in school lessons-time. On the other hand, during unstructured periods like SR time, results were the opposite. Younger students were more active than older students when there was no control by any expert, that is, when the PA only depended on the will of the participants in any kind of PA. ${ }^{21,41}$ Finally, during AS period no differences were found between ages in the current study. This could be explained by the high percentage of participation in organized sport activities, which are the most important contribution activities for MVPA during the day, ${ }^{21}$ and probably again the directed and organized sport activities made the quantity of MVPA similar between age groups.

As discussed earlier, previous findings related to the influence of weight status on MVPA have been scarce and inconsistent. Results of the current study did not show differences in PE lessons and SR periods, but a significant difference in favor of overweight students in the AS time was confirmed. Moreover, no differences in the participation in organized sport activities were found regarding the weight status groups $\left(\chi^{2}{ }_{1}=0.125, P=.723\right.$; nonoverweight group, $81.9 \%$ participated in organized sports activities and $18.1 \%$ did not; overweight group, $83.8 \%$ participated in organized sports activities and $16.2 \%$ did not). In line with our results, no differences were found between children with different weight status in previous studies during PE classes ${ }^{26}$ or SR time. ${ }^{5,26}$ However, the study of Kobel et $\mathrm{al}^{46}$ with German schoolchildren detected higher levels of habitual MVPA in overweight than nonoverweight students. The study by Hohepa et $\mathrm{al}^{7}$ with New Zealand adolescents also found that those who were classified as overweight/obese were more likely to be active than the normal weight adolescents. In addition, parental influences (eg, support, level of education, or their level of MVPA) or socioeconomic status could also be present in this result, as demonstrated previously. ${ }^{47}$ Therefore, further studies regarding this variable are necessary to clarify the complex relationship between weight status and MVPA in different contexts and countries.

The current study was focused in a school with particular characteristics of being allocated in the city center and situated in a middle-high socioeconomic neighborhood, but other schools (eg, rural or in outside neighborhoods) need to be studied and compared. This particularity will limit the generalization of the obtained results to the sample studied and similar contexts. Further studies with a different design (longitudinal or qualitative research) are also required to improve the knowledge about MVPA in adolescents.

\section{Acknowledgments}

We thank Aliisa Hatten for the English revision. Special thanks to the educational center, PE teacher, and students who participated in this study. DM-V is supported by a research grant from the Spanish Ministry of Education, Culture and Sport (AP2010-5905).

\section{References}

1. de Moraes AC, Guerra PH, Menezes PR. The worldwide prevalence of insufficient physical activity in adolescents; a systematic review. Nutr Hosp. 2013;28(3):575-584. PubMed

2. Cocca A, Liukkonen J, Mayorga-Vega D, Viciana J. Health-related physical activity levels in Spanish youth and young adults. Percept Mot Skills. 2014;118(1):247-260. PubMed doi:10.2466/10.06. PMS.118k16w1

3. Reiner M, Niermann C, Jekauc D, Woll A. Long-term health benefits of physical activity-a systematic review of longitudinal studies. BMC Public Health. 2013;13:813. PubMed doi:10.1186/1471-2458-13-813 
4. Stratton G. Physical activity levels of 12-13 year old schoolchildren during European handball lessons: gender and ability group differences. Eur Phys Educ Rev. 1996;2(2):165-173. doi:10.1177/1356336X9600200208

5. Ridgers ND, Salmon J, Parrish A, Stanley RM, Okely AD. Physical activity during school recess: a systematic review. Am J Prev Med. 2012;43(3):320-328. PubMed doi:10.1016/j.amepre.2012.05.019

6. Gao Z, Oh H, Sheng H. Middle school students' body mass index and physical activity levels in physical education. Res $Q$ Exerc Sport. 2011;82(1):145-150. PubMed doi:10.1080/02701367.2011.10599732

7. Hohepa M, Scragg R, Schofield G, Kolt GS, Schaaf D. Self-reported physical activity levels during a segmented school day in a large multiethnic sample of high school students. J Sci Med Sport. 2009;12(2):284-292. PubMed doi:10.1016/j.jsams.2007.11.005

8. Slingerland M, Borghouts LB, Hesselink MK. Physical activity energy expenditure in Dutch adolescents: contribution of active transport to school, physical education, and leisure time activities. J Sch Health. 2012;82(5):225-232. PubMed doi:10.1111/j.1746-1561.2012.00691.x

9. World Health Organization. Global Recommendation on Physical Activity for Health. Geneva, Switzerland: WHO; 2010.

10. United States Department of Health and Human Services. Strategies to Improve the Quality of Physical Education. Washington, DC: Centers for Disease Control and Prevention; 2010.

11. Kretschmann R. Objective measurement of physical activity levels in everyday physical education. Res Q Exerc Sport. 2014;85(1):143.

12. Dudley DA, Okely A, Cotton W, Pearson P, Caputi P. Physical activity levels and movement skill instruction in secondary school physical education. J Sci Med Sport. 2012;15(3):231-237. PubMed doi:10.1016/j.jsams.2011.10.005

13. Marmeleira J, Aldeias N, Da Graca P. Physical activity levels in Portuguese high school physical education. Eur Phys Educ Rev. 2012;18(2):191-204. doi:10.1177/1356336X12440022

14. Raustorp A, Boldemann C, Johansson M, Mårtensson F. Objectively measured physical activity level during a physical education class: a pilot study with Swedish youth. Int J Adolesc Med Health. 2010;22(4):469-476. PubMed doi:10.1515/IJAMH.2010.22.4.469

15. Brusseau TA, Kulinna PH. An examination of four traditional school physical activity models on children's steps counts and MVPA. Res Q Exerc Sport. 2015;86(1):88-93. PubMed doi:10.1080/02701367.2 014.977431

16. Ridgers ND, Saint-Maurice PF, Welk GJ, Siahpush M, Huberty J. Differences in physical activity during school recess. J Sch Health. 2011;81(9):545-551. PubMed doi:10.1111/j.1746-1561.2011.00625.x

17. Ridgers ND, Stratton G, Fairclough SJ. Assessing physical activity during recess using accelerometry. Prev Med. 2005;41(1):102-107. PubMed doi:10.1016/j.ypmed.2004.10.023

18. Silva P, Sousa M, Sá C, Ribeiro J, Mota J. Physical activity in high school during 'free-time'. Eur Phys Educ Rev. 2015;21(2):135-148. doi:10.1177/1356336X14555295

19. Ministry of Education and Culture. Basic Education Act 27.11.1992/1174/§23. Helsinki: Ministry of Education and Culture; 1992.

20. Lee H, Tamminen KA, Clark AM, Slater L, Spence JC, Holt NL. A meta-study of qualitative research examining determinants of children's independent active free play. Int J Behav Nutr Phys Act. 2015;12:5.. PubMed 10.1186/s12966-015-0165-9

21. Arundell L, Ridgers ND, Veitch J, Salmon J, Hinkley T, Timperio A. 5-year changes in afterschool physical activity and sedentary behavior. Am J Prev Med. 2013;44:605-611. PubMed doi:10.1016/j. amepre.2013.01.029

22. Silva P, Sousa M, Aires L, et al. Physical activity patterns in Portuguese adolescents: the contribution of extracurricular sports. Eur Phys Educ Rev. 2010;16(2):171-181. doi:10.1177/1356336X10381305
23. Trost SG, Rosenkranz RR, Dzewaltowski D. Physical activity levels among children attending after-school programs. Med Sci Sports Exerc. 2008;40:622-629. PubMed doi:10.1249/MSS.0b013e318161eaa5

24. Martínez-Gómez D, Veiga OL, Zapatera B, Gomez-Martinez S, Martinez D, Marcos A. Physical activity during high school recess in Spanish adolescents: the AFINOS study. J Phys Act Health. 2014;11(6):1194-1201. PubMed doi:10.1123/jpah.2012-0345

25. Hesketh K, Graham M, Waters E. Children's after-school activity: associations with weight status and family circumstance. Pediatr Exerc Sci. 2008;20:84-94. PubMed doi:10.1123/pes.20.1.84

26. Mayorga-Vega D, Viciana J. Differences in physical activity levels in school-based contexts: influence of gender, age, and weight status. Kinesiology. 2015;47(2):151-158.

27. Santos-Lozano A, Santín-Medeiros F, Cardon G, et al. Actigraph GT3X: validation and determination of physical activity intensity cut points. Int J Sports Med. 2013;34(11):975-982. PubMed doi:10.1055/s-0033-1337945

28. Calahorro F, Torres-Luque G, López-Fernández I, Santos-Lozano A, Garatachea N, Álvarez E. Actividad física y acelerometría; orientaciones metodológicas, recomendaciones y patrones. Nutr Hosp. 2015;31(1):115-128.

29. Trost SG, Loprinzi PD, Moore R, Pfeiffer KA. Comparison of accelerometer cut points for predicting activity intensity in youth. Med Sci Sports Exerc. 2011;43(7):1360-1368. PubMed doi:10.1249/ MSS.0b013e318206476e

30. Cole TJ, Bellizzi MC, Flegal KM, Dietz WH. Establishing a standard definition for child overweight and obesity worldwide: international survey. BMJ. 2000;320(7244):1240-1243. PubMed doi:10.1136/ bmj.320.7244.1240

31. Camliguney AF, Mengutay S, Pehlivan A. Differences in physical activity levels in 8-10 year-old girls who attend physical education classes only and those who also regularly perform extracurricular sports activities. Procedia Soc Behav Sci. 2012;46:4708-4712 doi:10.1016/j.sbspro.2012.06.323.

32. D'Haese S, Van Dyck D, Bourdeaughuij I, Deforche B, Cardon G. Organizing "Play Streets" during school vacations can increase physical activity and decrease sedentary time in children. Int J Behav Nutr Phys Act. 2015;12(14):1-9. PubMed 10.1186/s12966-015-0171-y

33. Lonsdale C, Rosenkranz RR, Peralta LR, Bennie A, Fahey P, Lubans DR. A systematic review and meta-analysis of intervention designed to increase moderate-to-vigorous physical activity in school physical education lessons. Prev Med. 2013;56:152-161. PubMed doi:10.1016/j. ypmed.2012.12.004

34. Andersen HB, Klinker CD, Toftager M, Pawlowki CS, Schipperijn J. Objectively measured differences in physical activity in five types of schoolyard area. Landsc Urban Plan. 2015;134:83-92. doi:10.1016/j. landurbplan.2014.10.005

35. Watson A, Eliott J, Mehta K. Perceived barriers and facilitators to participation in physical activity during the school lunch break for girls aged 12-13 years. Eur Phys Educ Rev. 2015;21(2):257-271. doi:10.1177/1356336X14567545

36. Uys M, Draper CE, Hendricks S, et al. Factors of influencing breaktime physical activity of South African primary school learners from low-income communities. J Phys Act Health. 2015;12(5):618-627. PubMed doi:10.1123/jpah.2013-0188

37. Perlman D. Influence of the Sport Education Model on amotivated students' in-class physical activity. Eur Phys Educ Rev. 2012;18(3):335345. doi:10.1177/1356336X12450795

38. Brooke HL, Corder K, Atkin A, van Sluijs E. A systematic literature review with meta-analyses of within- and between-day differences in objectively measured physical activity in school-aged children. Sports Med.2014;44(10):1427-1438. PubMed doi:10.1007/s40279-014-0215-5 
39. Cooper AR, Page AS, Wheeler BW, Hillsdon M, Griew P, Jago R. Patterns of GPS measured time outdoors after school and objective physical activity in English children: the PEACH project. Int J Behav Nutr Phys Act. 2010;7(1):31. PubMed doi:10.1186/1479-5868-7-31

40. Dumith SC, Gigante DP, Domingues MR, Kohl HW. Physical activity change during adolescence: a systematic review and a pooled analysis. Int J Epidemiol. 2011;40(3):685-698. PubMed doi:10.1093/ije/ dyq 272

41. Sahlqvist S, Goodman A, Cooper AR, Ogilvie D. Change in active travel and changes in recreational and total physical activity in adults: longitudinal findings from the iConnect study. Int J Behav Nutr Phys Act. 2013;10(1):28. PubMed doi:10.1186/1479-5868-10-28

42. Gao Z, Chen S, Stodden DF. A comparison of children's physical activity levels in physical education, recess, and exergaming. $J$ Phys Act Health. 2015;12(3):349-354. PubMed doi:10.1123/jpah.2013-0392

43. Plotnikoff RC, Costigan SA, Karunamuni ND, Lubans DR. Communitiy-based physical activity interventions for treatment of type 2 diabetes: a systematic review with meta-analysis. Front Endocrinol. 2013;29(4):3.
44. Beets MW, Shah R, Weaver RG, Huberty J, Beighle A, Moore JB. Physical activity in after-school programs: comparison with physical activity policies. J Phys Act Health. 2015;12:1-7. PubMed doi:10.1123/jpah.2013-0135

45. Brooke H, Atkin A, Corder K, Ekelund U, van Sluijs E. Changes in time-segment specific physical activity between ages 10 and 14 years: a longitudinal observational study. J Sci Med Sport. 2016;19:29-34. doi:10.1016/j.jsams.2014.10.003

46. Kobel S, Kettner S, Kesztyüs D, Erkelenz N, Drenowatz C, Steinacker JM. Correlates of habitual physical activity and organized sports in German primary school children. Public Health. 2015;129:237-243. PubMed doi:10.1016/j.puhe.2014.12.002

47. Jiménez-Pavón D, Fernández-Alvira JM, te Velde SJ, et al. Associations of parental education and parental physical activity (PA) with children's PA: The ENERGY cross-sectional study. Prev Med. 2012;55:310-314 doi:10.1016/j.ypmed.2012.07.011. PubMed 